\title{
Síndrome do Overlap: Esclerose sistêmica sem esclerodermia associado à polimiosite: Relato de Caso
}

\author{
Overlap Syndrome: Systemic Sclerosis without Polymyositis-Associated Scleroderma: \\ Case Report \\ Sindrome del Sobrelap: Esclerosis sistémica sin esclerodermia asociado a polimiositis: \\ Relato de Caso
}

Carla Daniele Nascimento Pontes ${ }^{1}$, Yuri José Almeida da Silva ${ }^{1}$, Rodrigo Bona Maneschy ${ }^{1}$, Lucianna Serfaty de Holanda ${ }^{1}$, Carolina Tavares Carvalho ${ }^{1 *}$, José Deorilo Cruz Gouveia dos Santos Junior ${ }^{1}$, Valeria da Silva Campos ${ }^{1}$, Mariana Diniz Araújo ${ }^{1,}$ Karen Larissa Lucena de Moraes ${ }^{1}$, Pâmela de Fátima Cunha Mendes de Lima' ${ }^{1}$.

\section{RESUMO}

Objetivo: O presente estudo teve como objetivo descrever a associação simultânea de duas doenças do tecido conjuntivo, de natureza auto-imune, sendo compostas pela esclerose sistêmica (ES) e polimiosite (PM). Demonstrando a raridade da sobreposição e importância de inclui- lá como hipótese diagnostica desses pacientes reumatológicos. Detalhamento do caso: Paciente do sexo masculino, 39 anos, com diagnóstico prévio de fibrose pulmonar idiopática, com presença de dispneia aos esforços, fenômeno de Raynaud, artralgia e fraqueza muscular proximal. Sendo encontrada a presença de marcadores reumatológicos e enzimas musculares positivos. Como o fator antinuclear (FAN) 1/320 padrão: misto do tipo nuclear pontilhado e nucleolar, ANTI-SCL $70 \geq 240$ e elevação na aldolase/creatina fosfoquinase. Considerações finais: Destaca-se a necessidade de novos estudos epidemiológicos e a realização de protocolos específicos para uma melhor abordagem diagnóstica e terapêutica desses tipos de casos, pois a relação da esclerose sistêmica e polimiosite, representa uma das síndromes de sobreposição mais incomuns na literatura.

Palavras-chave: Esclerose Sistêmica, Polimiosite, Doenças raras.

\section{ABSTRACT}

Objective: The present study aimed to describe the simultaneous association of two connective tissue diseases, of an autoimmune nature, being composed of systemic sclerosis (ES) and polymyositis (PM). Demonstrating the rarity of the overlap and the importance of including it as a diagnostic hypothesis for these rheumatological patients. Details of the case: Male patient, 39 years old, with previous diagnosis of idiopathic pulmonary fibrosis, with dyspnea on exertion, Raynaud's phenomenon, arthralgia and proximal muscle weakness. The presence of positive rheumatological markers and muscle enzymes was found. As standard antinuclear factor (ANA) 1/320: dotted and nucleolar nuclear type mixed, ANTI-SCL $70 \geq 240$ and elevation in aldolase / creatine phosphokinase. Final considerations: The need for further epidemiological studies and the performance of specific protocols for a better diagnostic and therapeutic approach for these types of cases, since the relationship between systemic sclerosis and polymyositis represents one of the most unusual overlapping syndromes in the literature.

Keywords: Systemic Sclerosis, Polymyositis, Rare deseases.

1 Fundação Santa Casa de Misericórdia do Pará, Belém - PA. *E-mail: caroltc94@hotmail.com 


\section{RESUMEN}

Objetivo: El presente estudio tuvo como objetivo describir la asociación simultánea de dos enfermedades del tejido conectivo, de naturaleza autoinmune, que se componen de esclerosis sistémica (ES) y polimiositis (PM). Demostrando la rareza de la superposición y la importancia de incluirla como hipótesis diagnóstica para estos pacientes reumatológicos. Detalles del caso: paciente masculino, de 39 años, con diagnóstico previo de fibrosis pulmonar idiopática, con disnea de esfuerzo, fenómeno de Raynaud, artralgia y debilidad muscular proximal. Se encontró la presencia de marcadores reumatológicos positivos y enzimas musculares. Como factor antinuclear estándar (ANA) 1/320: punteado y nucleolar de tipo nuclear mixto, ANTI-SCL $70 \geq 240$ y elevación de aldolasa / creatina fosfoquinasa Consideraciones finales: La necesidad de más estudios epidemiológicos y el rendimiento de protocolos específicos para un mejor enfoque diagnóstico y terapéutico para este tipo de casos, ya que la relación entre la esclerosis sistémica y la polimiositis representa uno de los síndromes superpuestos más inusuales en la literatura.

Palabras clave: Esclerosis Sistémica, Polimiositis, Enfermedades raras.

\section{INTRODUÇÃO}

As Síndromes de Overlap são raras e caracterizam-se pela presença simultânea de duas ou mais doenças do tecido conjuntivo, de natureza auto-imune. Os pacientes que estiverem nessa categoria devem preencher critérios diagnósticos separadamente para cada tipo de acometimento reumatológico. Foram descritos na literatura a associação de várias doenças, como esclerodermia com polimiosite e dermatomiosite, lúpus eritematoso sistêmico, artrite reumatoide e síndrome de Sjögren (PARENTE J, et al., 2011; LOPES D e MARTINS J, 2014). E essa sobreposição acaba dificultando ainda mais o diagnóstico precoce nesses pacientes. A esclerose sistêmica (ES) é uma doença sistêmica autoimune, sendo caracterizada, por três processos fisiopatológicos distintos: injúria vascular, autoimunidade (celular e humoral) e fibrose tecidual, levando a lesões em diversos órgãos internos, como pulmão, coração e trato gastrintestinal, bem como a manifestações musculoesqueléticas. Ainda não possui etiologia completamente definida, porém, define-se como causa multifatorial, possivelmente desencadeada por fatores ambientais em indivíduo geneticamente predisposto (CORAL-ALVARADO PC, et al., 2009; BEYER C, et al., 2009).

O envolvimento do sistema imune, tanto humoral quanto celular, está envolvido em todas as etapas do mecanismo patogênico, sendo a fibrose a mais representativa. A extensão da fibrose cutânea está associada à maior mortalidade na ES, pela produção de colágeno a partir dos fibroblastos. Os fibroblastos são ativados por moléculas produzidas pelas células $T$ e, também, pelos níveis baixos de oxigênio, ou por seus radicais, produzidos durante as crises de espasmos vasculares, ocorridas no Fenômeno de Raynaud (ZIMMERMANN AF e PIZZICHINI MMM, 2013). Essa doença ocorre em todas as áreas geográficas do mundo, em diversas raças e a incidência anual é estimada em 19:1.000.000 habitantes no mundo. No Brasil, ainda não existem dados epidemiológicos sobre a doença. As mulheres são acometidas três vezes mais que os homens e a idade média de aparecimento da doença é entre 30 e 50 anos (SAMARA AM, 2004).

Por ser uma doença heterogênea com amplo espectro clínico, a esclerose sistêmica é, de forma geral, classificada em ES difusa (com espessamento cutâneo grave e difuso), ES limitada (espessamento cutâneo limitado às extremidades distais e à face) e ES sem escleroderma ou sine escleroderma (espessamento cutâneo ausente). Este último, por não apresentar o sinal mais característico da ES, é considerado uma doença de difícil diagnóstico (APENZELLER S, et al., 2004). Apesar de o termo ter sido proposto desde Rodnan GP e Fennel RH (1962), apenas nos últimos anos as características clínicas e demográficas da ES sem esclerodermia foram mais bem definidas. Esta constitui uma rara apresentação, quando há envolvimento visceral importante, além de alterações vasculares e sorológicas características da ES (POORMOGHIM H, et al., 2000). Por sua vez, a polimiosite (PM) também está incluída no rol de doenças autoimunes, constituindo uma miopatia inflamatória sistêmica associada à alta morbidade e incapacidade funcional. É caracterizada por fraqueza simétrica na musculatura proximal de início subagudo, associada a 
alterações articulares, renais, cardiorrespiratórias e gastrointestinais (THEILACKER LR, et al., 2015; BARROS TBM, et al., 2014). Dessa forma, podemos inclui-la como diagnostico diferencial de outras colagenases, como a própria esclerose.

Em determinados quadros clínicos, observa-se a presença de sinais e sintomas semelhantes em relação as duas. Entre eles, encontramos o fenômeno de Raynaud e mialgia e, menos comumente, o acometimento visceral, com queixas respiratórias e relacionadas ao trato gastrointestinal (THEILACKER LR, et al., 2015). Disfagia, distúrbios cardíacos, acometimento respiratório, vasculite e calcificações subcutâneas (calcinoses) são manifestações extra musculares possíveis (DANKO K, et al., 2004). Essa miopatia está relacionada à imunidade celular e concentrações elevadas de interleucinas pró-inflamatórias: fator de necrose tumoral (TNF, interleucinas (IL-1e IL-6). Além disso, muitos autoanticorpos antinucleares e citoplasmáticos são encontrados nos pacientes com polimiosite. No entanto, a maioria desses anticorpos são inespecíficos, sendo comumente encontrado em outras desordens reumáticas (SHINJO SK, et al., 2013; BROUWER R, et al., 2001).

Devem ser levados em consideração pelo menos 2 critérios para o seu possível diagnostico: fraqueza muscular proximal das cinturas pélvica e escapular; presença de miosite à biópsia muscular; elevação de qualquer enzima muscular sérica: creatinofosfoquinase (CPK), sendo essa mais sensível, ou aldolase ou desidrogenase lática (DHL); eletromiografia compatível com miopatia e lesões cutâneas características de dermatomiosite: heliótropo; pápulas/ sinal de Gottron. Dessa forma, ambas as doenças são raras e de alta relevância na prática médica, pois possuem algumas similitudes em sua patogênese podendo haver sobreposição entre elas. O presente estudo teve como objetivo descrever a associação simultânea de duas doenças do tecido conjuntivo, de natureza auto-imune, sendo compostas pela esclerose sistêmica (ES) e polimiosite (PM). Demonstrando a importância da inclusão das superposições clínicas, nos diagnósticos diferenciais dos pacientes reumatológicos.

\section{DETALHAMENTO DO CASO}

Deu entrada, em 22/03/2018, paciente do sexo masculino, 39 anos, proveniente do ambulatório de pneumologia da Fundação Santa Casa de Misericórdia no Pará (FSCMPA), deu entrada na enfermaria São Roque com diagnóstico de fibrose pulmonar evoluindo há vários meses com quadro de dispnéia aos médios esforços, tosse seca, fraqueza muscular proximal, artralgia, presença do fenômeno de Raynaud em extremidades distais, astenia e perda ponderal de aproximadamente $30 \mathrm{kgs}$ em um ano.

Paciente apresentava como antecedentes pessoais asma, ex-tabagista (1 carteira de cigarro/dia por 5 anos), ex-etilista (aos finais de semana) e história ocupacional em garimpo por 8 anos. Nega alergias e uso de medicação de uso contínuo. No exame físico, paciente encontrava-se consciente, orientado em tempo e espaço, eupneico, afebril, acianótico, anictérico, mucosas normocoradas, níveis pressóricos estáveis, sem gânglios palpáveis, diurese e função intestinal presente, sono e apetite preservados. Ausculta cardíaca e ausculta pulmonar sem alterações, abdômen plano, normotenso, indolor a palpação, sem megalias e ruídos hidroaéreos presentes, descompressão brusca negativa e membros inferiores com pulsos, sem edemas e panturrilhas livres.

Foram solicitados exames laboratoriais, perfil do FAN, fator reumatoide e sorologias. Os dois primeiros testes são importantes para considerar o diagnóstico e prognóstico de distúrbios autoimune. O FAN resulta do reconhecimento de antígenos nucleares, nucleolares, citoplasmáticos, por auto-anticorpos presentes no soro do paciente. E o fator reumatoide é um auto-anticorpo, da classe IgM, dirigida contra IgG, normalmente associada a artrite reumatoide. Além de baciloscopia do escarro, com amostras negativas, ecocardiograma e tomografia computadorizada de tórax. Sendo iniciado ceftriaxone e prednisona $40 \mathrm{mg}$.

A tomografia computadoriza de tórax evidenciou a presença de micronodulos centrolobulares, associados a algumas opacidades ramificadas, com aspecto de arvore em brotamento, predominando nos lobos superiores e segmentos posteriores dos lobos inferiores. Espessamento irregular de septos 
interlobulares, sobretudo nos lobos inferiores. (constava entre os diagnósticos diferenciais: processo infeccioso granulomatoso). Bandas parenquimatosas e bronquiectasias/bronquioloectasias de tração nos segmentos posteriores dos lobos superiores, lobo médio e lobos inferiores, associados à área de consolidação/atelectasia. Ectasia do tronco da artéria pulmonar, medindo $3,7 \mathrm{~cm}$. Múltiplas linfonodomegalias calcificadas em cadeias paratraqueais, paraesofagicas, subaorticas, subcarinais e hilares, medindo ate $3,3 \mathrm{~cm}$. O diagnóstico pelas as alterações pulmonares associadas à linfonodomegalias, poderiam estar relacionadas a quadro de sarcoidose, com processo infeccioso granulomatoso em atividade.

Ainda durante o curso da internação, o paciente relatou alteração visual com diminuição da acuidade, febre, além de manter o quadro de artralgia. A tomografia computadorizada de crânio evidenciou acentuação dos sulcos da convexidade e cisternas basais, com dilatação compensatória do sistema ventricular. Áreas hipoatenuantes, dispersas pela substância branca peri-ventricular e sub-cortical sugestivas de microangiopatia.

Posteriormente, reajustada a dose de prednisona para $60 \mathrm{mg}$, com boa resposta clínica. Neste dia foram liberados os resultados negativos para o fator reumatoide e sorologia para hepatite B. Os resultando dos exames de FAN, aldolase e anti-escleroderma ANTI-SCL, foram liberados no dia 10/04/2018 mostrando respectivamente FAN 1/320 padrão: misto do tipo nuclear pontilhado e nucleolar, positivo para núcleo, nucléolo e placa metafásica com região organizadora do nucléolo, aldolase: 11(Referência: até 7,6 U/L), creatina fosfoquinase (CPK): 934 (Referência em homens: Homens: 22,0 a 334,0 U/L) e ANTI-SCL 70 240 (Referência: negativo).

Durante a avaliação da reumatologia em 11/04/2018 foram solicitados anti-centromero e antiribonucleoproteínas (ANTI-RNP), DNA nativo ou de dupla hélice (ANTI-DNA), além de marcadores de outras doenças do colágeno. No dia seguinte, o paciente realizou avaliação oftalmológica, onde se evidenciou no exame da fundoscopia: boa midríase, meios claros, retina aplicada, macula livre, sem foco exsudativo, disco óptico fisiológico. Não foi possível avaliar segmento anterior, pois havia a possibilidade de haver catarata subcapsular posterior em ambos os olhos (olho esquerdo> olho direito). Orientou-se encaminhamento para ambulatório de oftalmologia após alta e iniciou-se albendazol para realizar imunossupressão com ciclofosfamida, sendo prescritos pela equipe de reumatologia. No dia 13/04/2018, foram liberados os resultados negativos dos anticorpos anteriores. Com o resultado da hemocultura que mostrava Acinetobacter Baumanni Complex, foi iniciado moxifloxacino. Ainda no decorrer da internação, surgiram lesões tipo vesículas com base eritematosa, pruriginosas e dolorosas, na região dorso-lombar em faixa, pela qual foi prescrito aciclovir. No decorrer da mesma semana, o medicamento moxifloxacino foi substituído pelo imipenem.

O resultado da broncoscopia foi liberado no dia 16/04/2018 mostrando laringe: estruturas supra glóticas integras e de coloração normal. Ausência de estase salivar. Traqueia: calibre e direção preservados. Mucosa integra e de coloração normal. Carina principal: afilada, mucosa integra, coloração normal, arvore brônquica direita: brônquio fonte, superior, lingular e inferior, segmentares e subsegmentares, apresentamse revestidos por mucosa integra, coloração normal, concluindo que o exame estava sem anormalidades. Lavado com cocos gram positivos em cadeias frequentes. Escalonou-se então, o esquema vancomicina, imipenem e aciclovir, o paciente obteve uma boa resposta ao novo esquema.

Após 40 dias de internação e término dos esquemas de medicação com controle do quadro, o paciente obteve alta hospitalar, encaminhado para continuidade do acompanhamento via ambulatorial, com clínica médica e reumatologia.

\section{DISCUSSÃO}

A esclerose sistêmica sem esclerodermia diferencia-se das demais formas clínicas da doença, por apresentar somente acometimento visceral, ou seja, de órgãos internos. Não havendo envolvimento cutâneo. Ela representa uma entidade rara, com frequência de $2-8 \%$ na população mundial. E possui manifestações sistêmicas na seguinte ordem de prevalência: gastrointestinais (53-86\%), pulmonares (25- 
$75 \%$ ) e renais (2,5-3,7\%) (VERA-LASTRA O, et al., 2016) (GRAÑA D, et al., 2018). Apesar de a doença ser mais frequente em mulheres do que em homens, em média 3-8:1 e pico de incidência entre 45 e 64 anos (BASTOS et al, 2016), o presente estudo apresentava um paciente do sexo masculino, na faixa etária de 39 anos. Demonstrando dados divergentes no predomínio de gênero encontrado na literatura (HORIMOTO AMC E DA COSTA IP, 2014).

A forma visceral está associada à hipomotilidade esofágica, fibrose intersticial pulmonar, hipertensão pulmonar isolada, a lesões vasculares e alterações na capilaroscopia periungueal. Manifestações cardíacas (miocardioesclerose) e renais (crise renal esclerodérmica) não são frequentes nesses pacientes em questão. Sendo mais encontradas na esclerose difusa (APPENZELLER S, et al, 2004). No caso relatado, o paciente possuía diagnóstico prévio de fibrose pulmonar e desenvolveu inicialmente crise de dispneia a médios esforços, tosse seca, presença do fenômeno de Raynaud em extremidades distais, astenia e perda ponderal acentuada. E posteriormente, evoluiu com artralgia e fraqueza muscular proximal, sem edema ou eritema nessas regiões.

Anteriormente foram designados alguns critérios diagnósticos para a esclerose sistêmica sem esclerodermia. Dentre eles encontram-se a presença do fenômeno de Raynaud, juntamente com um acometimento visceral, como a hipomotilidade esofágica, além de fibrose ou hipertensão pulmonar primaria, e positividade laboratorial do FAN (SAMPAIO-BARROS PD, et al, 2013). Durante a investigação obtivemos os seguintes resultados: FAN 1/320 padrão: misto do tipo nuclear pontilhado e nucleolar, positivo para núcleo, nucléolo e placa metafásica com região organizadora do nucléolo, ANTI-SCL $70 \geq 240$ e elevação na aldolase/creatina fosfoquinase. A tomografia computadoriza de tórax que evidenciou micronódulos centrolobulares, algumas opacidades ramificadas e outras modificações estruturais. Nesses pacientes o FAN está presente em mais de $95 \%$ dos casos, independentemente da forma clínica da doença. E o anticorpo antitopoisomerase-1 (antitopo-1 ou anti-Scl-70), encontra-se em ate $20 \%-30 \%$ dos pacientes esclerodérmicos, sendo mais associado à forma difusa da esclerose sistêmica, e, corresponde a maior gravidade (MULLER CS, et al, 2011).

O envolvimento pulmonar da esclerose sistêmica é bastante diversificado, observa-se inflamação e fibrose intersticial com depósito excessivo da matriz extracelular e obliteração vascular. Podendo aparecer como hipertensão pulmonar, infiltrados intersticiais, micronódulos, fibroses pleurais e pneumonias aspirativas. Ele tende a surgir, em geral, dentro dos três primeiros anos do início da doença sistêmica e o seu achado aumenta a morbimortalidade destes pacientes (GUIDOLIN F, et al, 2005) (BASTOS AL, et al, 2016). Além disso, destaca-se também a história de fraqueza muscular proximal com elevação sérica de enzimas musculares, descritas anteriormente. Traduzindo um provável diagnóstico concomitante com polimiosite.

Segundo Bohan e Peter, os critérios de classificação ainda incluem electromiografia com alterações miopáticas, alterações características na biópsia muscular com ausência de sinais histológicos de outras doenças, para confirmação definitiva (HASMUCRAI D, et al, 2010). A sintomatologia clínica e os resultados dos exames complementares colaboraram para elucidação da base etiológica do caso em estudo. Tratandose de uma sobreposição de um quadro de esclerodermia e polimiosite, com envolvimento pulmonar. Um anticorpo dirigido contra o antígeno nucleolar PM-Scl é o principal marcador biológico desta doença. Estando presente em mais de $50 \%$ dos adultos com essa síndrome e persiste independentemente da sua atividade. Geralmente apresenta um curso moderado e que responde a imunossupressores e corticosteróides orais (TOLL A, et al, 2004).

O plano terapêutico está baseado em estratégias órgão-específicas, já que cada manifestação clínica pode precisar de uma abordagem individualizada. E na sequência, foi instituída terapia com prednisolona 40 $\mathrm{mg} /$ dia e que posteriormente sofreu reajuste para $60 \mathrm{mg} / \mathrm{dia}$ e imunossupressão com ciclofosfamida, com resposta eficiente. A literatura demonstra que pacientes que apresentam a manifestação de pneumopatia intersticial rapidamente progressiva, possuem uma resposta importante, com melhora na tolerância ao exercício e na própria função pulmonar, com doses semelhantes a empregada no caso (SAMPAIOBARROS PD, et al, 2013). 
Demais estudos propunham o uso do metotrexato como uma alternativa de tratamento, uma vez que tal medicamento tem se mostrado benéfico nas duas doenças em questão. Entretanto, tais autores não levaram em consideração a possibilidade de pneumonite por esta droga, ocasionando dificuldades para 0 seguimento clínico (BANDT M, et al, 1997).

Dessa forma, o relato estudado demonstra que a relação da esclerose sistêmica e polimiosite, é uma das síndromes de sobreposição mais incomuns. E que se destaca a necessidade de novos estudos epidemiológicos e a realização de protocolos específicos para uma melhor abordagem diagnóstica e terapêutica. Evitando assim a sua progressão para complicações mais graves e proporcionando qualidade de vida para esses pacientes.

\section{REFERÊNCIAS}

1. APPENZELLER S, et al. Crise renal como manifestação inicial de esclerose sistêmica sine scleroderma. Rev. Bras. Reumatol., São Paulo, 2004; 44(1): 87-89.

2. BANDT M, et al. Systemic sclerosis andsarcoidosis, a report of five cases. Br J Rheumatol,1997; 36:117-119.

3. BARROS TBM, et al. Nefropatia por IgA e polimiosite: uma rara associação. Rev. Bras. Reumatol, São Paulo, 2014; 54 (3): 231-233.

4. BASTOS AL, et al. Padrões tomográficos da doença pulmonar na esclerose sistêmica. Radiol Bras. 2016 ; 49 (5):316-321.

5. BEYER C, et al. Hypoxia in the pathogenesis of systemic sclerosis. Arthritis Research \& Therapy, $2009 ; 11$ (2): 220-229.

6. BROUWER R, et al. Autoantibody profiles in the sera of European patients with myositis. Ann Rheum Dis, 2001; 60 (2): 116-123.

7. CORAL -ALVARADO PC, et al. Systemic sclerosis: A world wide global analysis. Clin Rheumatol, $2009 ; 28$ (7): 757-765.

8. DANKO K, et al. Long-term survival of patients with idiopathic inflammatory myopathies according to clinical features: a longitudinal study of 162 cases. Medicine (Baltimore). 2004; 83 (1): 35-42.

9. GRAÑA D, et al. Esclerosis sistémica: forma de presentación y manejo terapéutico. Experiencia de un grupo de trabajo en Enfermedades Autoinmunes Sistémicas. Rev. Urug. Med. Interna. 2018; (1): 15-22.

10. GUIDOLIN F, et, al. Esclerose Sistêmica e Sarcoidose. Rev Bras Reumatol, 2005; 45 (5): 335-338.

11. HASMUCRAI D, et al. Envolvimento pulmonar na polimiosite. Rev Portug de Pneumo, 2010; 16 (4): $671-677$.

12. HORIMOTO AMC, DA COSTA IP. Sobreposição de esclerose sistêmica e artrite reumatoide: uma entidade clínica distinta? Ver. Bras. Reumatol., 2016; 5 (6): 287-298.

13. LOPES D, MARTINS J. Síndrome de Overlap - um caso clínico. Galicia Clin., 2014; 75 (3): 145-147.

14. MULLER CS, et al. Perfil de autoanticorpos e correlação clínica em um grupo de pacientes com esclerose sistêmica na região sul do Brasil. Rev Bras Reumato., 2011; 51 (4): 314-324.

15. PARENTE J, et al. SÍNDROMA DE OVERLAP. Acta Med Port., 2011; 24: 719-724.

16. POORMOGHIM H. Systemic Sclerosis Sine Scleroderma. Arthritis Rheum., 2000; 43 (2): 444-451.

17. RODNAN GP, FENNEL RH. Progressive systemic scleroderma sine scleroderma. JAMA, 1962; 180: 665-70.

18. SAMARA AM. Esclerose sistêmica. Rev. Bras. Reumatol., São Paulo, 2004; 44 (1): 09-10.

19. SAMPAIO-BARROS, P.D et al, Recomendações sobre diagnóstico e tratamento da esclerose Sistêmica. Rev. Bras Reumatol., 2013; 53 (3): 258-275.

20. SHINJO SK, et al. Dermatomiosite e polimiosite: da imunopatologia à imunoterapia (imunobiológicos). Rev. Bras. Reumatol., São Paulo, 2013; 53 (1): 105-110.

21. THEILACKER LR, et al. Síndrome Antissintetase: relato de dois casos e revisão da literatura. Rev. Bras. Reumatol., São Paulo, 2015; 55 (2): 177-180.

22. TOLL A, et al. Sclerodermatomyositis associated with severe arthritis. Dermatol Online J., 2004; 10 (2): 18.

23. VERA-LASTRA O, et al. Esclerosis sistémica sin esclerodermia en pacientes mexicanos. Serie de casos. Reumatol Clin., 2016: 1-3.

24. ZIMMERMANN AF, PIZZICHINI MMM. Atualização na etiopatogênese da esclerose sistêmica. Revista Brasileira de Reumatologia, 2013; 53 (6). 